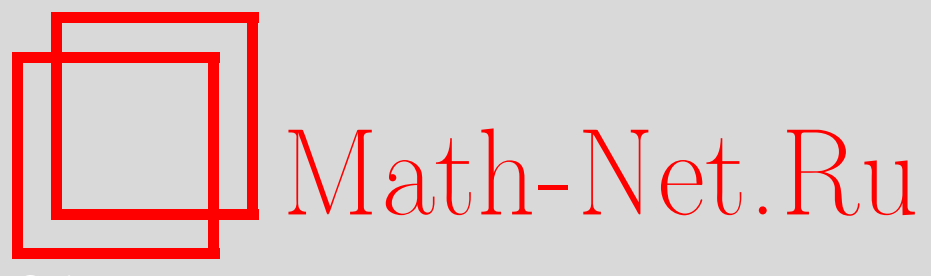

Д. В. Горбачев, М. В. Захарова, Об одной экстремальной задаче для периодических функций с малым носителем, Матем. заметки, 2006, том 80, выпуск 6, 940-942

DOI: https://doi.org/10.4213/mzm3370

Использование Общероссийского математического портала Math-Net.Ru подразумевает, что вы прочитали и согласны с пользовательским соглашением http://www.mathnet.ru/rus/agreement

Параметры загрузки:

IP : 34.229 .45 .116

26 апреля 2023 г., 13:58:09

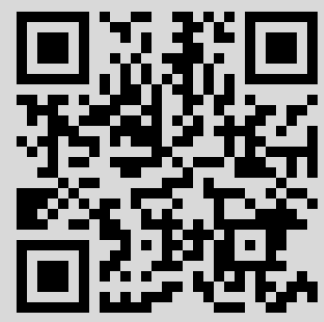




\section{ОБ ОДНОЙ ЭКСТРЕМАЛЬНОЙ ЗАДАЧЕ ДЛЯ ПЕРИОДИЧЕСКИХ ФУНКЦИЙ С МАЛЫМ НОСИТЕЛЕМ}

\section{Д. В. Горбачев, М. В. Захарова}

Пусть $0<h \leqslant 1 / 2, K(h)$ - класс непрерывных периодических четных действительных функций $f(x)=\sum_{n=0}^{\infty} a_{n} \cos (2 \pi n x)$, для которых $\sum_{n=0}^{\infty}\left|a_{n}\right|=1, \quad f(x) \equiv 0$ при $h \leqslant$ $|x| \leqslant 1 / 2$.

Рассматривается экстремальная задача нахождения величины

$$
B(h)=\sup _{f \in K(h)} a_{0}=\sup _{f \in K(h)} \int_{-h}^{h} f(x) d x .
$$

Эта задача и ее варианты имеют приложения в теории функций, аналитической теории чисел, цифровой обработке сигналов (см. [1]-[3]), в которых важно знать величину $B(h)$ для отдельных значений $h$. Ранее она была вычислена для $h=1 / 2,1 / 3,1 / 4,1 / 5,2 / 5$ (см. [3]). Здесь рассматривается случай $h=1 / 6$.

Пусть $\Gamma(x)$ - гамма-функция, $\mathrm{B}(x, y)=\Gamma(x) \Gamma(y) / \Gamma(x+y)$ - бета-функция [4],

$$
\gamma(z)=\frac{\mathrm{B}(4 / 3,4 / 3)}{\mathrm{B}((4-z) / 3,(4+z) / 3)}=\frac{\Gamma^{2}(4 / 3)}{\Gamma((4-z) / 3) \Gamma((4+z) / 3)}, \quad \gamma(0)=1 .
$$

Из равенства [4]

$$
\int_{0}^{\pi / 2} \cos ^{a} t \cos (b t) d t=\frac{\pi}{2^{a+1}} \frac{\Gamma(1+a)}{\Gamma(1+(a+b) / 2) \Gamma(1+(a-b) / 2)},
$$

следует, что

$$
\gamma(z)=A \int_{-1 / 6}^{1 / 6} \cos ^{2 / 3}(3 \pi x) \cos (2 \pi z x) d x, \quad A=5 \cdot 2^{2 / 3} \mathrm{~B}\left(\frac{4}{3}, \frac{4}{3}\right)>0 .
$$

Teоpema. Для $h=1 / 6$

$$
\mathrm{B}\left(\frac{1}{6}\right)=\frac{1}{2+(20 / 3) \mathrm{B}(4 / 3,4 / 3)}=0.1807247 \ldots .
$$

Экстремальная функиия имеет вид

$$
f^{*}(x)=A B \cos ^{2 / 3}(3 \pi x), \quad|x| \leqslant \frac{1}{6}, \quad a_{0}^{*}=B, \quad a_{n}^{*}=2 B \gamma(n), \quad B=B\left(\frac{1}{6}\right) .
$$

ДоказАтеЛЬСтво. Пусть $f \in K(1 / 6)$. Оценим коэффициент $a_{0}$ сверху.

Из определения класса $K$ имеем

$$
\begin{gathered}
I_{1}=0=-\frac{4}{3} f\left(\frac{1}{6}\right)+\frac{1}{3} f\left(\frac{1}{2}\right)=\sum_{n=0}^{\infty} \alpha(n) a_{n}, \\
\alpha(n)=-\frac{4 \cos (\pi n / 3)}{3}+\frac{\cos (\pi n)}{3}=\left\{\begin{array}{rl}
-1, & n=0,1,5,6, \\
1, & n=2,3,4,
\end{array} \quad \alpha(n+6)=\alpha(n) .\right.
\end{gathered}
$$

Работа выполнена при поддержке Российского фонда фундаментальных исследований, гранты № 05-01-39005 и № 06-01-00372. 
Пусть

$$
\beta(z)=\frac{\sin \pi z}{\pi z\left(1-z^{2}\right) \gamma(z)}, \quad z \in \mathbb{C} .
$$

Установим некоторые свойства функций $\beta$ и $\gamma$. Из представления (2) следует, что функция $\gamma$ является четной целой функцией экспоненциального типа $2 \pi / 6=\pi / 3$, а из (1) и свойств гамма-функции [4] для нее получаем бесконечное произведение

$$
\gamma(z)=\prod_{k=1}^{\infty}\left(1-\frac{z^{2}}{(3 k+1)^{2}}\right)=\prod_{i \in I}^{\infty}\left(1-\frac{z^{2}}{i^{2}}\right), \quad I=\{4,7, \ldots, 3 i+1, \ldots\} .
$$

Отсюда и из (5) получаем, что функция $\beta$ является четной целой функцией экспоненциального типа $\pi-\pi / 3=2 \pi / 3$ с бесконечным произведением

$$
\begin{gathered}
\beta(z)=\frac{\prod_{k=1}^{\infty}\left(1-z^{2} / k^{2}\right)}{\left(1-z^{2}\right) \prod_{k=1}^{\infty}\left(1-z^{2} /(3 k+1)^{2}\right)}=\prod_{i \in I^{\prime}}\left(1-\frac{z^{2}}{i^{2}}\right), \\
I^{\prime}=\{2,3, \ldots, k, \ldots\} \backslash I=\{2,3,5,6, \ldots, 3 i+2,3 i+3, \ldots\} .
\end{gathered}
$$

При больших $z>0$ [4]

$$
\frac{\Gamma(z+a)}{\Gamma(z+b)}=z^{a-b}\left(1+\frac{1}{2 z}(a-b)(a+b-1)+O\left(z^{-2}\right)\right) .
$$

Отсюда и из тождества $\Gamma(z) \Gamma(1-z)=\pi / \sin \pi z$ получаем, что при больших $z>0$

$$
\beta(z)=\frac{z^{2 / 3} \sin \pi z}{3^{5 / 3} \Gamma^{2}(4 / 3)\left(z^{2}-1\right) \sin \pi((z-4) / 3)}\left(1+O\left(z^{-2}\right)\right)=O\left(z^{-4 / 3}\right), \quad \gamma(z)=O\left(z^{-5 / 3}\right) .
$$

Таким образом, $\beta \in L_{1}(\mathbb{R}) \cap L_{2}(\mathbb{R})$. Отсюда и по теореме Пэли-Винера имеем

$$
\begin{aligned}
& \widehat{\beta}(x)=\int_{\mathbb{R}} \beta(z) e^{-2 \pi i z x} d z=\int_{\mathbb{R}} \beta(z) \cos (2 \pi z x) d z \in C(\mathbb{R}), \\
& \widehat{\beta}(x) \equiv 0, \quad|x| \geqslant \frac{1}{3}, \quad \beta(z)=2 \int_{-1 / 3}^{1 / 3} \widehat{\beta}(x) \cos (2 \pi z x) d x
\end{aligned}
$$

При $z>0$ из (1) и (5) следует, что

$$
\begin{aligned}
\frac{\beta(z)}{\beta(z+3)} & =\frac{(z+2)(z+3)(z+4) \gamma(z+3)}{z(1-z)(z+1) \gamma(z)}=\frac{(z+2)(z+3)(z+4)(1-z)}{z(1-z)(z+1)(z+4)} \\
& =\frac{(z+2)(z+3)}{z(z+1)}>1
\end{aligned}
$$

т.е. $\beta(z)>\beta(z+3)$. Отсюда и из (7) получаем цепочку неравенств

$$
\beta(1)>\beta(4)>\beta(7)>\cdots>\beta(3 i+1)>\cdots>0 .
$$

Необходимые свойства функций $\beta$ и $\gamma$ установлены. Продолжим оценку $a_{0}$. Из (8) и определения класса $K(1 / 6)$ следует, что

$$
\begin{gathered}
I_{2}=2 b \int_{0}^{1 / 2} f(x) \widehat{\beta}\left(x-\frac{1}{2}\right) d x=2 b\left(\int_{0}^{1 / 6}+\int_{1 / 6}^{1 / 2}\right)=0 \\
b=-\frac{2}{\beta(1)}=-\frac{1}{\gamma(1)}=-\frac{20 \mathrm{~B}(4 / 3,4 / 3)}{3}<0 .
\end{gathered}
$$


Подставляя сюда разложение функции $f$ и учитывая (8), получаем, что

$$
\begin{aligned}
0=I_{2} & =2 b \sum_{n=0}^{\infty} a_{n} \int_{0}^{1 / 2} \widehat{\beta}\left(x-\frac{1}{2}\right) \cos (2 \pi n x) d x \\
& =b \sum_{n=0}^{\infty} a_{n} \cos (\pi n) \int_{-1 / 2}^{1 / 2} \widehat{\beta}(x) \cos (2 \pi n x) d x=b \sum_{n=0}^{\infty}(-1)^{n} a_{n} \beta(n) .
\end{aligned}
$$

Из (4), (10) и (11) находим, что

$$
0=I_{1}+I_{2}=\sum_{n=0}^{\infty} a_{n} \omega(n), \quad \omega(n)=\alpha(n)+(-1)^{n} b \beta(n)=\alpha(n)-\frac{2(-1)^{n} \beta(n)}{\beta(1)} .
$$

Покажем, что

$$
|\omega(n)| \leqslant 1, \quad n \in \mathbb{N} .
$$

В силу (6) и (7) имеем $\mathbb{N}=\{1\} \cup I \cup I^{\prime}$. Из (4) и (12) $\omega(1)=1$. При $n \in I^{\prime}$ в силу (7) и (4) $|\omega(n)|=|\alpha(n)|=1$. Пусть $n=3 i+1 \in I$, где $i \in \mathbb{N}$. Тогда, учитывая (4), получаем, что

$$
\omega(n)=\alpha(3 i+1)+\frac{2(-1)^{i} \beta(3 i+1)}{\beta(1)}=\left\{\begin{array}{cl}
-1+\frac{2 \beta(3 i+1)}{\beta(1)}, & i \text { четное, } \\
1-\frac{2 \beta(3 i+1)}{\beta(1)}, & i \text { нечетное. }
\end{array}\right.
$$

Отсюда и из (9) следует, что $|\omega(n)| \leqslant 1$ для $n \in I$. Неравенство (13) установлено.

Вернемся к оценке $a_{0}$. Из (12), свойств класса $K(1 / 6)$ и (10) находим

$$
\begin{gathered}
a_{0} \omega(0)=-\sum_{n=1}^{\infty} a_{n} \omega(n), \quad\left|a_{0} \omega(0)\right| \leqslant \sum_{n=1}^{\infty}\left|a_{n}\right|=1-\left|a_{0}\right|, \\
\omega(0)=\alpha(0)+b \beta(0)=-1-\frac{20}{3} \mathrm{~B}\left(\frac{4}{3}, \frac{4}{3}\right)<0, \\
a_{0} \leqslant\left|a_{0}\right| \leqslant \frac{1}{1+|\omega(0)|}=\left(2+\frac{20}{3} \mathrm{~B}\left(\frac{4}{3}, \frac{4}{3}\right)\right)^{-1}=B .
\end{gathered}
$$

Эта оценка правильная и достигается на функции $f^{*} \in K(1 / 6)$ из (3). Для нее выполняется равенство (12) и в силу (3), (4), (6), (7), (12), (14)

$$
\left|a_{n}^{*}\right|=a_{n}^{*} \omega(n), \quad n \in \mathbb{N}, \quad a_{0}^{*} \omega(0)=-\sum_{n=1}^{\infty}\left|a_{n}^{*}\right|, \quad \sum_{n=0}^{\infty}\left|a_{n}^{*}\right|=1, \quad a_{0}^{*}=B .
$$

Теорема доказана.

Постановка задачи и предположение о виде экстремальной функции принадлежит Д. В. Горбачеву; М. В. Захаровой выполнено строгое доказательство теоремы.

\section{СПИСОК ЦИТИРОВАННОЙ ЛИТЕРАТУРЫ}

[1] S. Konyagin, I. Shparlinski, Character Sums with Exponential Functions and their Applications, Cambridge Univ. Press, Cambridge, 1999. [2] Н. Н. Андреев, С. В. Конягин, А. Ю. Попов, Матем. заметки, 60:3 (1996), 323-332. [3] Д. В. Горбачев, Избранные задачи теории функций и теории приближений и их приложения, Изд-во ТулГУ, Тула, 2004. [4] Г. Бейтмен, А. Эрдейи, Высшие трансцендентные функиии, 1, Наука, М., 1965.

Д. В. Горбачев, М. В. Захарова

Поступило

Тульский государственный университет 16.05.2006

E-mail: dvg@uic.tula.ru 